\title{
Biomarkers for Chronic Neuropathic Pain and their Potential Application in Spinal Cord Stimulation: A Review
}

\author{
Chibueze D. Nwagwu ${ }^{1}$; Christina Sarris, MD³; Yuan-Xiang Tao, PhD, MD²; and Antonios Mammis, $\mathrm{MD}^{1,2}$
}

Departments of ${ }^{1}$ Neurological Surgery and ${ }^{2}$ Anesthesiology, Rutgers New Jersey Medical School, Newark, New Jersey, USA; and ${ }^{3}$ Barrow Neurological Institute, Phoenix, Arizona, USA.

Summary: This review was focused on understanding which substances inside the human body, increase and decrease with increasing neuropathic pain. We reviewed various studies, and saw correlations between neuropathic pain and components of the immune system (this system defends the body against diseases and infections). Our findings will especially be useful for understanding ways to reduce or eliminate the discomfort, chronic neuropathic pain brings with it. Spinal cord stimulation (SCS) procedure is one of the few fairly efficient remedial treatments for pain. A follow-up study will apply our findings from this review to SCS, in order to understand the mechanism, and further optimize efficaciousness.

Key Words: spinal cord stimulation; pain biomarkers; chronic neuropathic pain; cytokines.

\section{$\underline{\text { Introduction }}$}

Chronic neuropathic pain disorders represent a common long-term disability in the United States, as well as, globally. They affect 1 in 4 Americans. Chronic neuropathic pain treatment has limited success because of poor understanding of the mechanisms that lead to the initiation and maintenance. Additionally, the effectiveness of neuropathic pain management regimens and procedures has been difficult to determine in the past, due to the subjectivity related to pain perception, and a lack of standardized assessment of neuropathic pain. However, one of the most effective management strategies in recent times is spinal cord stimulation (SCS). The main goals of spinal cord stimulation are to improve physical function and quality of life in the patients afflicted with chronic neuropathic pain associated with complex regional pain syndrome (CRPS), failed back syndrome, and other chronic neuropathic pain syndromes [1-2]. Despite limited knowledge of how people benefit from SCS, more than 20,000 stimulators are implanted each year, with more than a half-billion dollars in revenue [3]. While it is generally believed that spinal cord stimulation inhibits pain transmission in the dorsal horn, the exact mechanisms by which SCS relieves neuropathic pain is unknown. Chronic neuropathic pain is caused often by inflammation and/or nerve injury. The advances have shown that inflammation and nerve injury produce changes in the expression of cytokines, neurotransmitters, and structural proteins [4]. It is very likely that there are changes in the body's serum biomarkers of neuropathic pain before SCS and after SCS. Such a study would contribute greatly to the field of neuromodulation, as objective quantifiers of neuropathic pain control before and after SCS have not yet been investigated. Such definitive data regarding the effectiveness of SCS in relieving neuropathic pain and improving function will be important in future use of SCS.

In preparation for the launching of this study, the authors' goal with this transcript is to provide a review of the literature regarding known biomarkers for chronic neuropathic pain, and then prepare a role for biomarker analysis in the prediction of therapy success in SCS.

\section{$\underline{\text { Data }}$}

The expression of certain genes is altered under chronic pain conditions. This alteration has helped provide an insight into the identification of potential biomarkers [5]. Current advanced research suggests that genetic expression of cytokines, positively or negatively, correlates with the experience of chronic pain. This neg- 


\section{Table 1: Cytokines and hormones elevations in various studies}

\begin{tabular}{|c|c|c|c|}
\hline Cytokines/Chemokines & Pain & Diagonosis & References \\
\hline \multirow[t]{4}{*}{ TNF- $\alpha$} & \multirow[t]{4}{*}{ Increased } & Neuropathic Pain/CRPS & {$[6]$} \\
\hline & & Neuropathic Pain/CRPS & {$[17]$} \\
\hline & & Fibromyalgia & {$[18]$} \\
\hline & & Fibromyalgia & [19] \\
\hline \multirow[t]{2}{*}{ IL-1 $\beta$} & \multirow[t]{2}{*}{ Increased } & Tension Headache & {$[22]$} \\
\hline & & Neuropathic Pain/CRPS & {$[12]$} \\
\hline IL-2 & Increased & Neuropathic Pain/CRPS & {$[12]$} \\
\hline \multirow[t]{4}{*}{ IL-6 } & \multirow[t]{4}{*}{ Increased } & Fibromyalgia & [19] \\
\hline & & Neuropathic Pain/CRPS & {$[17]$} \\
\hline & & Tension Headache & {$[23]$} \\
\hline & & Chronic Constriction Injury & {$[13][15]$} \\
\hline \multirow[t]{4}{*}{ IL-8 } & \multirow[t]{4}{*}{ Increased } & Fibromyalgia & {$[18]$} \\
\hline & & Fibromyalgia & [19] \\
\hline & & Tension Headache & {$[25]$} \\
\hline & & & {$[24]$} \\
\hline IL-33 & Increased & Chronic Constriction Injury & [9] \\
\hline CXCL1 & Increased & Chronic Constriction Injury & {$[15]$} \\
\hline CCL3 & Increased & Chronic Constriction Injury & [7] \\
\hline CCR5 & Increased & Chronic Constriction Injury & {$[7]$} \\
\hline \multirow[t]{5}{*}{ IL-10 } & \multirow[t]{5}{*}{ Decreased } & Neuropathic Pain/CRPS & {$[20]$} \\
\hline & & Neuropathic Pain/CRPS & {$[12]$} \\
\hline & & Fibromyalgia & {$[18]$} \\
\hline & & Fibromyalgia & [19] \\
\hline & & & {$[24]$} \\
\hline \multirow[t]{2}{*}{ IL-4 } & \multirow[t]{2}{*}{ Decreased } & CRPS & {$[10]$} \\
\hline & & Neuropathic Pain & {$[10]$} \\
\hline
\end{tabular}

ative or positive correlation primarily depends on the nature of the cytokine. Cytokines are signaling proteins which mediate the activation, differentiation, and proliferation of immune cells. They can be pro-inflammatory or anti-inflammatory. A misaligned balance between pro-inflammatory and anti-inflammatory cytokines has been common in most of the studies conducted (Table 1). Pro-inflammatory cytokines such as IL-1 $\beta$, IL-6, IL-2, IL-33, CCL3, CXCL1, CCR5, and TNF- $\alpha$, have been found to play significant roles in the amplification of chronic pain states. In studies involving discogenic pain, Complete Freund's adjuvant (CFA)-induced discogenic pain in animal models has been observed to coincide with a sustained up-regulation of the above named cytokines [6]. In a more recent study, chronic constriction injury (CCI)-induced rats (neuropathic pain induction) were shown to have increased serum levels of CCL3 and CCR5. Even more interesting, an intrathecal injection of the anti-inflammatory cytokine, IL-4, and the CCL3-neutralizing antibody, reduced the CCI-induced neuropathic pain, estimated by a plantar test [7]. Other studies have also shown that the selective genetic impairment of the highlighted pro-inflammatory cytokines attenuated nerve-injury induced pain behavior, observed in neuropathic pain models [8]. Particularly, Zarpelon et al. revealed that CCI-induced rats showed reduced mechanical hyperalgesia when the IL-33 receptor gene, IL-33R (ST2), was knocked out, 
compared to the wild-type mice [9].

On the other hand, one study showed that blood levels of anti-inflammatory cytokines (such as IL-10 and IL-4) of Complex Regional Pain Syndrome (CRPS) patients were lower compared to the control [10]. A recent study also shows a distinction of the significant increases of pro-inflammatory cytokines based on the part of the back affected. There were more significant elevations ( $p<0.05$, student's t test) of pro-inflammatory cytokines in the plasma of lower back pain patients than in upper back patients, when compared to controls [11]. There has also been a study focusing on the levels of the aforementioned cytokines in patients with painful neuropathy in contrast with painless neuropathy and healthy control subjects. Patients with a painful neuropathy had about double the level of IL-2 expression $(p=0.001)$, TNF expression $(p<0.0001)$ and protein levels $(p=0.009)$ than the controls. The study further indicated there was about double the IL-2 and TNF level expression ( $p=0.03 ; p=0.001)$ and protein levels in painful neuropathy $(p=0.04 ; p=0.04)$ than patients with painless neuropathy. On the contrary, levels of mRNA expression of the anti-inflammatory cytokines, IL-10 and IL-4 were considerably lower in patients with painful neuropathy than in patients with painless neuropathy $(p=0.001)[12]$.

Several other studies, focused on the antagonist and agonist effects of some drugs targeting pro-inflammatory and anti-inflammatory cytokines, also have pointed out their significance with pain. Certain known analgesics were seen to reduce the levels of pro-inflammatory cytokines in the studies reviewed. There was a study about (CCI)-induced rats, in which case, this induced injury significantly, elevated the levels of pro-inflammatory cytokines, and decreased the serum levels of anti-inflammatory cytokines. Omeprazole, a known remedy for stomach pain, was observed to reduce the levels of pro-inflammatory cytokines (TNF- $\alpha$, IL-1 $\beta$, and IL-6) to normal, compared with the CCI control. It is important to note that this was while curbing the CCI-induced neuropathic pain, measured with the paw withdrawal latency [13]. Zhou et al. also highlighted the significance of certain drugs in determining the correlation between cytokines and neuropathic pain. CCI was again, used to induce neuropathic pain on rat models; and in turn, Paenoflorin, an established anal- gesic [14] was administered. Once Paenoflorin was introduced, significant decreases in serum levels of pro-inflammatory cytokines of CCI-induced rats (IL$1 \beta$, IL-6, TNF- $\alpha$, and CXCL1) were observed, compared to the CCI-control [15]. The cytokines identified here, are the ones that showed correlation in various studies reviewed.

Even though cytokines are the key chronic pain biomarkers, according to the studies reviewed, there are still other proteins and nucleotides that have been observed to associate with chronic pain disorder. Two studies laid emphasis on regulatory microRNAs (miRNAs), which are small non-coding RNA molecules involved in post-transcriptional gene regulation. miRNAs achieve this by binding to mRNAs and degrading them or repressing their functions. Orlova et al. showed that $60 \%$ of CRPS patients in their study showed a significant down-regulation of 18 different miRNAs. The rest of the patients, however, showed variable (contradicting) miRNA levels. miRNA levels show variability, depending on the gene being regulated [5]. Tao et al. revealed that an increased inflammatory stimulation by the cytokine IL- $1 \beta$ in normal and osteoarthritis chondrocytes produced a significant down-regulation of the miRNA, miR-558, and a significant up-regulation of miR-21 in DRG neurons. A connection between IL-1 $\beta$ and miR-21 was attributed to AP-1, which is a transcription factor located in the promoter site of the mRNA, and is activated by IL-1 $\beta$ [4]. siRNAs have the same features as miRNAs, in the sense, that they are regulatory nucleotides. They also show variability, depending on the gene being regulated. SIRT1, a deacetylase, functions in regulating various pathways, including inflammation. It was observed that an intrathecal injection of SRT170, an SIRT1 agonist, reduced serum levels of NF- $\kappa \mathrm{B}$, a transcription factor for pro-inflammatory cytokines, in CCI-induced rat models. When SRT170-siRNA (a regulator of the regulator) was administered before SRT170, there was no agonistic effect [16].

\section{$\underline{\text { Discussion }}$}

Chronic neuropathic pain affects a tremendous amount of the population. There are few effective therapies. However, outcomes are hard to determine due to the subjective nature of pain. We would like to devise a 
strategy that would establish objectivity of pain assessment. After review of various studies relating to pain biomarkers, we found that serum levels of pro-inflammatory cytokines and chemokines, such as IL-1 $\beta$, IL-6, IL-2, IL-33, CCL3, CXCL1, CCR5, and TNF- $\alpha$, were significantly up-regulated during chronic pain experience. On the other hand, anti-inflammatory cytokines such as IL-10 and IL-4 were found to show significant down-regulation during chronic pain state. Regulatory miRNAs, siRNAs, and deacetylases that coincide with these cytokines, also showed negative correlation, corresponding to the cytokine they were regulating.

The authors would like to apply this knowledge to SCS, a therapy for chronic neuropathic pain, in an attempt to develop a biomarker profile to help predict success. This study will be a prospective study including patients scheduled for SCS. One month before SCS surgery, patients will complete a survey assessing their subjective level of pain on the visual analog scale and subjective level of function. Patients will also have venipuncture performed, and serum will be analyzed for levels of pain biomarkers. After SCS surgery, patients will be followed at 6 more time points: 2 weeks, 1 month, 3 months, 6 months, 1 year, and 2 years. At each time point, the survey will be re-administered and blood work will be repeated. By evaluating patients pre-operatively and post-operatively, we will be able to assess subjective and objective levels of pain, allowing us to analyze trends in pain biomarkers in the context of patient-reported pain measurement. The duration of this study will be 4 years. Each subject will participate in this study for a total period of 25 months, which will allow us to follow these patients for 2 years postoperatively.

\section{$\underline{\text { Conclusion }}$}

The review of various studies relating to inflammationand/or nerve injury-induced chronic pain disorder led us to hypothesize that the application of the spinal cord stimulation procedure should relatively reduce serum pro-inflammatory cytokines, and relatively increase serum levels of anti-inflammatory cytokines. This should, in turn, help us understand the mechanism of spinal cord stimulation, thereby optimizing the efficaciousness of the procedure, and perhaps allow us to make predictions regarding therapy success. A follow-up prospective study regarding serum biomarker profile in SCS patients is currently being undertaken.

\section{Author Disclosure}

The authors declare no conflicts of interest.

\section{Disclosure of Funding}

This work was supported by grants from the University of Medicine and Dentistry of New Jersey and the National Institutes of Health, Bethesda, Maryland (grant numbers: NS072206, HL117684, and DA033390).

Corresponding Authors: Chibueze D. Nwagwu, MD and Antonois Mammis, MD. Emails:

dominicnwagwu@gmail.com; mammisan@njms. rutgers.edu.

Editor: Renyu Liu, MD; PhD; Associate Professor, irector of Preoperative Medicine, Department of Anesthesiology and Critical Care; erelman School of Medicine at the University of Pennsylvania, 336 John Morgan building, 3620 Hamilton Walk, Philadelphia, PA 19104 . Phone: 2157461485; FAX: 2153495078 liur@uphs.upenn.edu

\section{Additional publication details}

Journal short name: Transl Perioper \& Pain Med

Received Date: August 16, 2016

Accepted Date: September 26, 2016

Published Date: September 28, 2016

Transl Perioper \& Pain Med 2016; 1(3):33-38

Citation and Copyright

Citation: Nwagwu CD, Sarris C, Tao YX, Mammis A. T. Biomarkers for Chronic Neuropathic Pain and their Potential Application in Spinal Cord Stimulation: A Review. Transl Perioper \& Pain Med 2016; 1(3): 33-38

Copyright: (C) 2016 Nwagwu CD, et al. This is an open-access article distributed under the terms of the Creative Commons Attribution License, which permits unrestricted use, distribution, and reproduction in any medium, provided the original author and source are credited. 


\section{References}

1. Lee AW, Pilitsis JG. Spinal Cord Stimulation: Indications and Outcomes. Neurosurg. Focus 2006; 21:1-6. PMID: 17341047.

2. Kumar K, Hunter G, Demeria D. Spinal Cord Stimulation in Treatment of Chronic Benign Pain: Challenges in Treatment Planning and Present Status, a 22-year Experience. Neurosurgery 2006; 58:481-491. PMID: 16528188.

3. Vallejo R, Kramer J, Benjamin R. Neuromodulation of the Cervical Spinal Cord in the Treatment of Chronic Intractable Neck and Upper Extremity Pain: A Case Series and Review of the Literature. Pain Physician 2007; 10:305311. PMID: 17387353.

4. Lutz BM, BekkerA, Tao YX. Non-coding RNAs: New Players in Chronic Pain. Anesthesiology 2014; DOI: 10.1097/ALN.0000000000000265. PMID: 24739997.

5. Orlova IA, Alexander GM, Qureshi RA, Sacan A, et al. MicroRNA Modulation in Complex Regional Pain Syndrome. Journal of Translational Medicine. 2011; 9:195. PMID: 22074333.

6. Jung WW, Kim HS, Shon JR, et al. Intervertebral Disc Degeneration-induced Expression of Pain-Related Molecules: Glial Cell-derived Neurotropic Factor as a Key Factor. J Neurosurg Anesthesiol 2011; 23: 329-334. PMID: 21659885.

7. Sun S, Chen D, Lin F, Chen M, Yu H, Hou L, Li C. Role of Interleukin-4, the Chemokine CCL3 and its Receptor CCR5 in Neuropathic Pain. Mol Immunol 2016; 77:184-192. PMID: 27522478.

8. Clark AK, Old EA, Malcangio M. Neuropathic Pain and Cytokines: Current Perspectives. Journal of Pain Research 2013; 6:803-814. PMID: 24294006.

9. Zarpelon et al. Spinal Cord Oligodendrocyte-derived Alarmin IL-33 mediates Neuropathic Pain. FASEB J 2016; 30(1): 54-65. PMID: 26310268.
10. Parkitny L, McAuley JH, Pietro FD, Stanton TR, O'Connell NE, Marinus J, van Hilten JJ, Moseley GL. Inflammation in Complex Regional Pain Syndrome. Neurology 2013; 80:106-117. PMID: 23267031.

11. Li Y, Liu J, Liu ZZ, Duan DP. Inflammation in Low Back may be detected from the Peripheral Blood: Suggestions for Biomarker. Bioscience Reports (2016) DOI: 10.1042/BSR20160187. PMID: 27380953.

12. Uceyler N, Rogausch JP, Toyka KV, Sommer C. Differential Expression of Cytokines in Painful and Painless Neuropathies. Neurology 2007; 69: 42-49. PMID: 17606879.

13. Chanchal SK, Mahajan UB, Siddharth S, Reddy N, Goyal SN, Patil PH, Bommanahalli BP, Kundu CN, Patil CR, Ojha S. In Vivo and In Vitro Protective Effects of Omeprazole against Neuropathic Pain. Sci Rep 2016; 6:30007. PMID: 27435304.

14. Yin D, Liu YY, Wang TX, Hu ZZ, Qu WM, Chen JF, Cheng NN, Huang ZL. Paeoniflorin exerts analgesic and hypnotic effects via adenosine A1 receptors in a mouse neuropathic pain model. Psychopharmacology (Berl). 2016 Jan; 233(2):281-93. PMID: 26514553.

15. Zhou J, Wang L, Wang J, Wang C, Yang Z, Wang C, Zhu Y, Zhang J. Evid Based Complement Alternat Med 2016; 2016:8082753. PMID: 27429639.

16. Lv C, Hu HY, Zhao L, Zheng H, Luo XZ, Zhang J. Intrathecal SRT1720, a SIRT1 agonist, exerts anti-hyperalgesic and anti-inflammatory effects on chronic constriction injury-induced neuropathic pain in rats. Int J Clin Exp Med 2015; 8(5): 7152-7159. PMID: 26221253

17. Kraychete DC et al. Serum Cytokine Levels in Patients with Chronic Low Back Pain due to Herniated Disc: Analytical Cross-Sectional Study. Sao Paulo Med J. 2010; 128(5): 259-292. PMID: 21181064. 
18. Bazzichi L et al. Cytokine Patterns in Fibromyalgia and Their Correlation with Clinical Manifestations. Clinical and Experimental Rheumatology 2007; 25:225-230. PMID: 17543146.

19. Iannuccelli $\mathrm{C}$ et al. Pathophysiology of Fibromyalgia: A Comparison with the Tension-Type Headache, a Localized Pain Syndrome. Ann. N.Y. Acad. Sci. 2010; 1193: 78-83. PMID: 20398011.

20. Backonja MM, Coe CL, Muller DA, Schell K. Altered Cytokine Levels in the Blood and Cerebrospinal Fluid of Chronic Pain Patients. Journal of Neuroimmunology 2008; 195: 157-163. PMID: 18325600.

21. Empl M1, Renaud S, Erne B, Fuhr P, Straube A, Schaeren-Wiemers N, Steck AJ. TNF-Alpha Expression in Painful and Non-painful Neuropathies. Neurology 2001; 56(10):1371-7. PMID: 11376190.

22. Della Vedova $C$ et al. Peripheral Interleukin-1 $\beta$ Levels are elevated in Chronic Tension-Type Headache Patients. Pain Res Manag 2013; 18(6):301-306. PMID: 23957020.

23. Koçer A et al. Interleukin-6 Levels in Tension Headache Patients. Clin J Pain 2010; 26: 690693. PMID: 20664340.

24. Bekker A, Haile M, Kline R, Didehvar S, Babu R, Martiniuk F, Urban M. The effect of intraoperative infusion of dexmedetomidine on the quality of recovery after major spinal surgery. J Neurosurg Anesthesiol. 2013; 25(1):16-24. PMID: 22824921.

25. Dominques RB, Duarte H, Rocha NP, Teixeira AL. Increased Serum Levels of Interleukin-8 in Patients with Tension-Type Headache. Cephalagia 2014; 0(0):1-6. PMID: 25416323. 